\title{
Oncological and functional outcomes of extraperitoneal laparoscopic radical prostatectomy: An 18-years, single-center experience
}

\author{
Francesco Saverio Grossi, Emanuele Utano, Paolo Minafra, Pier Paolo Prontera, Francesco Schiralli, \\ Antonio De Cillis, Evangelista Martinelli, Marco Lattarulo, Meri Luka, Antonio Carrieri, Angelo D'Elia \\ Urology Unit, S.S. Annunziata Hospital, Taranto, Italy.
}

\begin{abstract}
Summary Objective: To present a retrospective analysis on the oncological and functional outcomes of a single-center experience on a large series of extraperitoneal laparoscopic radical prostatectomies (eLRP) with an extended follow-up.

Materials and methods: Herein we present a retrospective review of patients who underwent eLRP. Oncological and functional follow-up data were collected by means of outpatient visits and telephone interviews, assessing overall mortality and biochemical recurrence-free survival. Patients with clinical T4 stage prostate cancer (PCa), previous surgery for benign prostatic hyperplasia (BPH), previous androgen deprivation, radiotherapy, concomitant chemotherapy and/or experimental therapies, and with insufficient follow-up data were excluded. Preoperative data recorded were age, body mass index, ultrasound prostate volume, preoperative PSA and clinical stage of PCa. Operative data (operative time, nerve sparing technique and any perioperative complication) and pathological findings were obtained by consulting the surgical and pathological reports. Oncological and functional follow-up were collected during follow-up visits and telephone interview.

Results: Between January 2001 and December 2019, overall 938 eLRP were performed at our Institution. The median follow-up was 132 months. $69.7 \%$ of the patients had complete dataset. The estimated overall biochemical recurrence (BCR)-free survival was $71.4 \%$ at 5 years and $58.9 \%$ at 10 years. Cancer specific survival was $84,5 \%$. Erectile function was preserved in the most of patients as postoperative IIEF-5 score within 12 months after surgery was $>12$ in the $82.1 \%$. About the urinary incontinence, $0.76 \%$ of the patients presented severe incontinence (continued and persistent loss of urine) and $7.0 \%$ were mildly incontinent (using up to one pad per day).

Conclusions; eLRP has shown oncological and functional results comparable to other minimally invasive techniques and to open radical prostatectomy (ORP), with favorable perioperative outcomes than the open technique and a reduced complication rate.
\end{abstract}

KEY WORDS: Prostate cancer; Radical prostatectomy; Laparoscopic prostatectomy; Laparoscopy; Extraperitoneal prostatectomy.

Submitted 27 May 2021; Accepted 27 June 2021

\section{INTRODUCTION}

Prostate cancer ( $\mathrm{PCa}$ ) represents the most commonly diagnosed cancer in men (1). Radical prostatectomy (RP) rep- resents a first-line option for the treatment of localized PCa (2). Open RP is the traditional approach, but it is burdened by higher perioperative morbidity, greater blood loss and longer hospitalization (3).

Over the last three decades, mini-invasive techniques have increasingly gained popularity owing to their advantages on perioperative outcomes over open RP. At present, about two every three RPs are laparoscopic or robotassisted $(4,5)$.

Laparoscopic radical prostatectomy (LRP) could be performed in either transperitoneal or extraperitoneal route. Both these approaches have pros and cons: the transperitoneal LRP (tLRP) provides a broader surgical space with full exposure of all the anatomical landmarks of the pelvis; on the other hand, the extraperitoneal laparoscopic radical prostatectomy (eLRP) resembles more the open retropubic RP (6). It also decreases the risk of anesthetic and surgical complications, since it avoids the exposure of intraperitoneal structures and requires less steep Trendelenburg tilt.

eLRP was first described by Raboy in 1997(7). Since then, no clear evidence of the superiority of one approach to LRP over the other has been highlighted. Nevertheless, data on very large series of eLRP with long follow-up are still missing.

This work presents a retrospective, long-term follow-up analysis of a single-center experience on a large series of eLRP, over an 18-years period.

\section{Material AND MEthods}

In this retrospective cohort study, we retrospectively reviewed data of 938 patients who underwent eLRP at our Institution between January 2001 and December 2019. Among these, 168 presented exclusion factors and were removed from the analysis while 113 were excluded because of insufficient follow-up or incomplete dataset available. All the procedures were performed by three different surgeons (FSG, DB, and AD), using a standardized technique, as described below. All of them were already skilled in laparoscopic surgery, since they had already performed at least 20 laparoscopic procedures at the starting point of the analysis.

Exclusion criteria were: Clinical T4 stage prostate cancers, history of benign prostatic surgery, patient previously 
treated with androgen deprivation and/or radiotherapy, previous or concomitant chemotherapy and/or experimental therapies. Additionally, men with less than 6months follow-up and with largely incomplete dataset were excluded.

Preoperative data recorded were age, body mass index (BMI), ultrasound prostate volume, preoperative PSA, Gleason score, and clinical stage. Patients were classified in risk groups by considering preoperative clinical stage. Operative data (operative time, nerve sparing technique and any perioperative complication) and pathological findings were obtained by consulting the surgical and pathological reports. Oncological and functional followup data were collected by means of outpatient visits and telephone interviews, assessing overall mortality and biochemical recurrence-free survival (BRFS).

\section{Oncological management of patients after RP}

In most of the cases, men with pT2 or pT3 tumors or men with positive surgical margins followed a "wait and see" strategy with eventual subsequent salvage radiotherapy at PSA recurrence.

Biochemical recurrence (BCR) was defined as two consecutive values of PSA $\geq 0.2 \mathrm{ng} / \mathrm{mL}$ at least 6 weeks after surgery. This threshold matches the classical EAU definition of BCR after RP (8), even though this definition has recently changed (9). Patients having persistent PSA levels $>0.2 \mathrm{ng} / \mathrm{mL}$ were considered as having BCR, as well. Neither this group of patients, nor men who received adjuvant radiation therapy for locally advanced disease were excluded from the analysis.

\section{Functional outcomes}

Urinary incontinence (UI) was evaluated by the number of pads needed per $24 \mathrm{~h}$ and stratified as follows: 0 pad (no incontinence), 1 pad (mild incontinence), and $\geq 2$ pads (severe incontinence). International Index of Erectile Function-5 (IIEF-5) questionnaire were administered to evaluate the 12-month erectile function, as well as to assess pre-existing erectile dysfunction (10).

\section{Statistical analysis}

Statistical analysis was carried out with the software Stata MP15 (StataCorp LLC, College Station, TX, USA). Baseline data were analyzed using descriptive statistics: frequencies were expressed as percentages while continuous variablse were presented as medians and interquartile ranges. We considered a two-tailed p-value of $<0.05$ as statistically significant.

We present the following article in accordance with the STROBE reporting checklist.

\section{Surgical technique}

The eLRP technique adopted in our Urology department was already presented in another work (11). A laparoscopic radical prostatectomy with bilateral pelvic lymph node dissection was performed with an extraperitoneal laparoscopic approach, regardless Gleason score and clinical T-stage. The patient was placed in supine position, the table hyperextended at the pubic symphysis level.

A $20^{\circ}$ Trendelenburg tilt was given. The initial incision was medial, $1 \mathrm{~cm}$ below the umbilicus. After rectus fascia identification and incision, a blunt dissection was performed under direct vision with a round shape balloon to develop the extraperitoneal space of Retzius. Four trocars were then placed under direct control of the surgeon's index finger: two pararectal $10 \mathrm{~mm}$ trocars and two $5 \mathrm{~mm}$ trocars, $2 \mathrm{~cm}$ cranially and medially from the anterior superior iliac spine. A $10 \mathrm{~mm}$ structural balloon trocar served as the optical trocar in the median sub umbilical incision.

Bilateral extended pelvic lymph node dissection was first performed. The template for lymphadenectomy included common, internal and external iliac and obturator lymph nodes.

The bladder was divided from the prostatic base in a bladder neck-sparing fashion whenever it was possible. This step was completed using blunt dissection as much as possible. Seminal vesicles and deferent ducts were dissected and freed.

The dissection of the posterior surface of the prostate could be made along an intra-, inter-, or extrafascial plane according to risk stratification and preoperative erectile function. When a nerve-sparing procedure was planned, the Denonvilliers' fascia was incised in the midline and the dissection along the intrafascial plane was carried on towards the lateral surface of the prostate, strictly avoiding cautery and limiting stretching of the neurovascular bundles. The prostatic pedicles were controlled using endoscopic Hem-o-Lok clips and cut.

The dorsal venous complex (DVC) was divided using an ultrasonic energy scalpel or a combined bipolar/ultrasonic energy device. This is usually sufficient to control any bleeding from DVC and no stitch was usually required. Then the prostate apex was carefully divided from the membranous urethra to obtain the longest possible urethral stump. Thereafter, the urethra was cut with cold scissors.

The specimen was placed in a laparoscopic bag and removed through the sub umbilical incision. The incision was extended if needed. A classical double-running VLock 3-0 suture was adopted during the vesico-prostatic anastomosis.

Antibiotics, intravenous fluids, and prophylaxis for deep vein thrombosis were given per institutional protocol. Blood parameters, diuresis and drainage were monitored and the drain tube removed as soon as possible. The ambulation was encouraged, and diet was started on the first postoperative day.

The urethral catheter was removed after ten days, always after performing cystogram.

All patients were followed with standardized protocol. Outpatient visits were scheduled every 3-month for the first year. At each visit, a physical examination and PSA were routinely performed. The clinical history about continence and erectile function was collected. Sexual function was measured using the IIEF-5 questionnaire. Men reporting a daily use of no pad were considered as completely continent, whereas the use of one pad per day was considered as mild incontinence.

Patients were addressed to continence ad sexual rehabilitation with postoperative pelvic floor muscle training and PDE5 Inhibitors and/or intracavernous injections with customized protocol. 
Table 1.

Demographic and perioperative characteristics.

\begin{tabular}{|c|c|}
\hline$N=657$ & Results \\
\hline Age (y), median (IQR) & $67(62-73)$ \\
\hline BMI (kg/m²), median (IQR) & $28.3(26.7-29.5)$ \\
\hline $\begin{array}{l}\text { Prostate volume (m)), median (IQR) } \\
\text {. }\end{array}$ & $52.2(45.5-61.3)$ \\
\hline$\overline{P S A}(\mathrm{ng} / \mathrm{ml})$, median (IQR) & $10.5(6.4-14.2)$ \\
\hline Preoperative potency (IIEF-5> 12), n (\%) & $513(78.1)$ \\
\hline Preoperative urinary continence, $\mathrm{n}(\%)$ & $657(100)$ \\
\hline Operative time (m), median (IQR) & $115(85-184)$ \\
\hline$\overline{\text { Postoperative LOS (d), median (IQR) }}$ & $3.5(2.5-4)$ \\
\hline $\begin{array}{l}\text { Overall NS procedures, n (\%) } \\
\text { Yes } \\
\text { Bilateral } \\
\text { Monolateral } \\
\text { No }\end{array}$ & $\begin{array}{l}408(62.1) \\
243(37.0) \\
165(25.1) \\
249(37.9)\end{array}$ \\
\hline \multicolumn{2}{|c|}{$\begin{array}{l}\text { BMI: Body Mass; PSA: Prostate-Specific Antigenn; IIEF-5: International Index of Erectile Function-5, } \\
\text { LOS: Inggth of stay; NS: nerve-sparing; IQR: Interquartile range. }\end{array}$} \\
\hline
\end{tabular}

Table 2.

Intraoperative and perioperative complications.

\begin{tabular}{|l|cl|}
\hline & N & (\%) \\
\hline Blood transfusion & 21 & 3.2 \\
\hline Bladder neck contracture & 6 & 0.9 \\
\hline Anastomotic leak & 9 & 1.4 \\
\hline Ochiepididimitis & 3 & 0.5 \\
\hline Symptomatic lymphocele & 12 & 1.8 \\
\hline Rectal injury & 3 & 0.5 \\
\hline Ileus & 8 & 1.2 \\
\hline Deep vein thrombosis & 4 & 0.6 \\
\hline Total adverse events & 66 & \\
\hline Clavien Dindo I-II & 53 & 8.1 \\
\hline Clavien Dindo II--V & 10 & 25 \\
\hline
\end{tabular}

\section{RESULTS}

Between 2001 and 2019, 938 patients with median age of 67 years (IQR: 62-73) years underwent eLRP at our Urology department and were followed with a median follow-up time of 132 months (IQR: 63-173 mo). Within the total population, $657(69.7 \%)$ have no exclusion criteria and had complete oncological and functional data. The median BMI of the patients was $28.3 \mathrm{~kg} / \mathrm{m}^{2}$ (IQR: 26.7-29.5). The median ultrasound prostate volume was 52.2 cc (IQR: 45.5-61.3). Preoperative median PSA value was $10.5 \mathrm{ng} / \mathrm{mL}$ (IQR: 6.4-14.2). Complete demographic and operative features are reported in Table 1.

The median operative time was 115 minutes (IQR: 85184 minutes), whereas the median length of postoperative hospitalization was 3.5 days (IQR: 2.5-4).

The posterolateral dissection of the prostate was carried out using a nerve-sparing technique in 408/657 patients (62.1\%), in $37.0 \%$ on both sides while in $25.1 \%$ monolaterally. The $37.9 \%$ of men did not receive a nerve-sparing surgery.

Overall, 66 perioperative complications occurred in 51 men (7.8\%). All complications with Clavien-Dindo classification are listed in Table 2.

At definitive histology, pathological stage was T2 in 535 (81.4\%), T3 in 117 (17.8\%) and T4 in 5 cases (0.8\%).
Lymph nodes invasion was observed in 57 men (8.7\%). Positive surgical margins (PSM) were found in 87 (13.2\%). Among men with T2 prostate cancer, 52 had PSM (9.7\%). 156 patients $(23.7 \%$ ) received immediate adjuvant radiation therapy for locally advanced disease (Table 3 ).

During the observation period, a total of 58 patients died. In nine of them, the cause of death was linked to PCa. The estimated overall BCR-free survival was $71.4 \%$ at 5 years and $58.9 \%$ at 10 years. In organ-confined prostate cancer the BCR-free survival was $77.2 \%$ at 5 years and $65.2 \%$ at 10 years. In pT3 stage the BCR-free survival was $47.9 \%$ at 5 years and $32.5 \%$ at 10 years (Table 3 ).

Preoperative IIEF-5 questionnaire showed a good erectile function or at most a mild-moderate erectile dysfunction in $78.1 \%$ of the cases (513/657). All these men were interested in postoperative resumption of sexual activity. Among these patients, respectively $56.9 \%$ and $28.4 \%$ underwent nerve-sparing prostatectomy.

Postoperative IIEF-5 score within 12 months after surgery was $>12$ in the $82.1 \%(421 / 513)$.

As for urinary incontinence, after a minimum of 12 months after the surgery, the great majority of patients experimented a complete recovery of the urinary continence (no needing for pad). On the other hand, 5 patients $(0.76 \%)$ presented severe incontinence (continued and persistent loss of urine) and 46 (7.0\%) were mildly incontinent (using one pad per day) (Table 4).

\section{Table 3.}

Pathological and oncological outcomes.

\begin{tabular}{|c|c|c|c|}
\hline$N=657$ & & N (\%) & \\
\hline pT2 & & $535(81.4)$ & \\
\hline$\overline{p T 3}$ & & $117(17.8)$ & \\
\hline$\overline{\text { pT4 }}$ & & $5(0.8)$ & \\
\hline$\overline{\mathrm{pN}+}$ & & $57(8.7)$ & \\
\hline Overall PSM & & 87 (13.2) & \\
\hline pT2 & & $52(9.7)$ & \\
\hline$\overline{p T 3}$ & & $32(27.4)$ & \\
\hline$\overline{\mathrm{pT} 4}$ & & $3(60)$ & \\
\hline Immediate adjuvant RT & & $156(23.7)$ & \\
\hline \multirow[t]{2}{*}{ Overall mortality } & & $58(8.9)$ & \\
\hline & 5 years & BCR free survival & 10 years \\
\hline Overall & 71.4 & & 58.9 \\
\hline$\overline{\mathrm{pT} 2}$ & 77.2 & & 65.2 \\
\hline$\overline{\mathrm{pT} 3}$ & 47.9 & & 32.5 \\
\hline
\end{tabular}

Table 4.

Functional outcomes 12 months after surgery.

\begin{tabular}{|l|c|}
\hline Postoperative recovery EF, $n(\%)$ & $421 / 513(82.1)$ \\
\hline Surgical technique ${ }^{*}, n(\%)$ & $292(56.9)$ \\
Bilateral NS & $146(28.5)$ \\
Monolateral NS & $75(14.6)$ \\
Non-NS & $606(92.2)$ \\
\hline Complete continence $^{* *}, n(\%)$ & $46(7)$ \\
\hline Mild incontinence ${ }^{* *}, n(\%)$ & $5(0.8)$ \\
\hline Severe incontinence ${ }^{* *}, n(\%)$ & \\
\hline BEF: erectile function; IIEF-5: International Index of Erectile Function-5; NS: nerve-sparing technique. \\
"In men with preoperative IIEF-5 $\geq 12$. \\
" Continence: $n$ pad; mild incontinence: 1 pad; severe incontinence $\geq 2$ pad. \\
\hline
\end{tabular}




\section{Discussion}

There is an ongoing debate about the worthiness of eLRP versus tLRP. Both the approaches share the main advantages of laparoscopy over open surgery, such as a better visualization of the surgical field, lower blood loss, a more precise and watertight anastomosis that allows early catheter removal, and a shorter hospital stay. For this reason, the widespread diffusion of minimally invasive prostatectomy have led to a significant reduction of the surgical burden, while ensuring similar oncological results and complication rates compare to open RP (12$14,34)$.

However, no evidence still exists about the superiority of one laparoscopic approach over the other in terms of perioperative outcomes and incidence of complications (15). The transperitoneal approach offers the best visibility and workspace for pelvic surgery. However, the extraperitoneal route reduces potential complications linked to the peritoneum opening, such as bowel injury, ileus, intraperitoneal bleeding or urinary leakage, and formation of intraperitoneal adhesions. Moreover, eLRP have gained popularity among urologists, since it seems a more straightforward procedure $(16,17)$. Some authors also suggests that this approach may shorten the learning curve, but this point remains controversial (18).

The rapidly increasing application of the mini-invasive techniques makes long-term data essential for a proper counselling of the patients. Many studies have been directed to show the results of laparoscopic radical extraperitoneal prostatectomy, some even with very large sample size (19). Nevertheless, insufficient data on large series of eLRP with long-term follow-up are available. With this study, we filled this gap by presenting the results of a large cohort of extraperitoneal laparoscopic radical prostatectomy with extended follow-up.

\section{Oncological outcomes}

The complete resection of the tumor is a primary goal of radical prostatectomy and the presence of PSM after RP is predictor of PSA recurrence and is considered a negative prognostic factor. In our population, the overall rate of PSM was $13.2 \%$, while it was $9.7 \%$ in the subgroup of $\mathrm{T} 2$ tumors. Such findings are consistent with those previously reported. The overall rate of positive surgical margins after laparoscopic prostatectomy varies from $19.2 \%$ to $38.6 \%$ (20-23). Very large series of eLRP reported PSM rates of $10.8-16.1 \%$ and $31.2-34.6 \%$ in pT2 and pT3 cancers, respectively $(19,24)$. However, comparative data between extraperitoneal and intraperitoneal LRP show no differences in terms of PSM $(25,26)$, as well as no significant difference exist between open and laparoscopic RP (27).

Regardless of the surgical technique, one of the most relevant predictors for PSM is tumor stage. However, analyzing data by groups, no difference are shown between open prostatectomy and mini-invasive techniques in PSM rate for T2 and T3 tumors (27).

Another important indicator of the oncological safety of a surgical approach is biochemical recurrence (BCR), which has been associated with increased mortality (28). In the present investigation, the overall BCR-free survival was $71.4 \%$ at 5 years and $58.9 \%$ at 10 years. Stratifying our population by tumor stage, it was $77.2 \%$ at 5 years and $65.2 \%$ at 10 years for T2 stage and $47.9 \%$ at 5 years and $32.5 \%$ at 10 years for T3 stage tumors (Table 3 ).

Of note, these results are slightly below those already presented in other studies. Indeed, after ORP, the 10years BCR-free survival rate is $80 \%$ for T2 prostate cancer (29) and 54\% for pT3 tumors (30); on the other hand, the overall 10-year BCR-free survival was $75.6 \%$ after LRP (31). Our results probably represent the result of the lack of patient selection and, above all, of the "wait and see" strategy adopted in the majority of cases instead of a more aggressive attitude with early initiation of adjuvant therapies.

Many papers have directly compared the incidence of BCR between LRP to ORP. To date, the oncological data with the largest follow-up (49 months) were provided in the work of Martinez-Holguin et al. (32). This work did not highlight any difference in the incidence of $\mathrm{BCR}$ between the two techniques. No long-term data on BCR exist on te direct comparison between tLRP and eLRP.

\section{Functional outcomes}

We classified patients in three groups: full continence (no need for diapers), mild incontinence (1 per day) and severe incontinence (more than 2 per day). After a twelve-month postoperative follow-up, $92.2 \%(n=606)$ of 657 patients were completely continent, $7 \%(n=46)$ had mild incontinence, and $0.76 \%(\mathrm{n}=5)$ needed more than 2 pads per day. These data are consistent with previously published results for $\operatorname{LRP}(19,31,35)$. Stolzenburg et al. presented continence results for the extraperitoneal approach reporting a 12 -months continence rate of $92 \%$. Overall, we offered a nerve-sparing eLRP to 408 patients $(62.1 \%)$, while, among patients who presented good preoperative erectile function, the $85.4 \%$ underwent nervesparing prostatectomy. In this subgroup, $82.1 \%$ of men preserved the erectile function 12 month after surgery. Our findings on erectile function recovery are in line with some data reported in other series of nerve-sparing prostatectomy, though potency rates after open nervesparing ORP vary considerably across studies, reaching up to $86 \%$ at 12 months in selected patients (33). In our work, patients are not the result of meticulous selection, except for the identification of the necessary requirements to indicate a nerve-sparing prostatectomy. However, a similar rate of continence recovery partly depends also on our definition of erection recovery, that includes men with mild-moderate erectile dysfunction (IIEF-5 score higher than 12).

\section{Perioperative outcomes}

In our series, eLRP was shown to be a procedure with short operative times, and a reduced incidence of intra and perioperative complications.

Different comparative cohort studies have reported that the extraperitoneal approach needs shorter times (16, 17), but data of a meta-analysis comparing perioperative results and complications of intraperitoneal and extraperitoneal RP show that eLRP and tLRP have similar operative times. Moreover, blood loss and rate of transfusion of the two techniques are comparable. No significant differences were observed for the rate of intraoperative 
complications and the rate of open conversion, whereas a higher rate of postoperative complications was reported in the tLRP group (25).

Several limitations of the present study have to be addressed: the single-centre retrospective design of the present analysis potentially represents a bias. Moreover, this a consecutive series of 657 patients who underwent eLRP over a period of 18 years by three different surgeons. In that way, this series reflects the evolution of this technique and of the surgeons' learning curve along such a long time. Moreover, the lack of a standardized protocol for the oncological management and the functional rehabilitation represents a limitation.

\section{Conclusions}

Extraperitoneal RP brings considerable advantages in terms of perioperative outcomes (short duration of surgery, reduced blood losses, low risk of complications) compared to other laparoscopic techniques and ORP. It also presents similar results in the main oncological objectives and with optimal recovery of continence and erectile function.

\section{REFERENCES}

1. Siegel RL, Miller KD, Jemal A. Cancer statistics, 2020. CA Cancer J Clin. 2020; 70(1):7-30.

2. https://uroweb.org/wp-content/uploads/EAU-EANM-ESTROESUR-ISUP-SIOG-Guidelines-on-Prostate-Cancer-2021V4.pdf

3. Ilic D, Evans SM, Allan CA, et al. Laparoscopic and robotic-assisted versus open radical prostatectomy for the treatment of localised prostate cancer. Cochrane Database Syst Rev. 2017; 9:CD009625.

4. Sujenthiran A, Nossiter J, Parry M, et al. National cohort study comparing severe medium-term urinary complications after robotassisted vs laparoscopic vs retropubic open radical prostatectomy. BJU Int. 2018; 121:445-52.

5. Hyldgård VB, Laursen KR, Poulsen J, Søgaard R. Robot-assisted surgery in a broader healthcare perspective: a difference-in-difference-based cost analysis of a national prostatectomy cohort. BMJ Open. 2017; 7:e015580.

6. Bollens $R$, Vanden Bossche $M$, Roumeguere $T$, et al. Extraperitoneal laparoscopic radical prostatectomy. Results after 50 cases. Eur Urol. 2001; 40:65-9

7. Raboy A, Ferzli G, Albert P. Initial experience with extraperitoneal endoscopic radical retropubic prostatectomy. Urology. 1997; 50:849-53

8. Boccon-Gibod L, Djavan W, Hammerer P, et al. Management of prostate-specific antigen relapse in prostate cancer: a European Consensus. Int J Clin Pract. 2004; 58:382-90.

9. Toussi A, Stewart-Merrill S, Boorjian S, et al. Standardizing the definition of biochemical recurrence after radical prostatectomy. What prostate specific antigen cut point best predicts a durable increase and subsequent systemic progression? J Urol. 2016; 195:1754-9.

10. Rhoden E, Telöken C, Sogari P, Vargas Souto C. The use of the simplified International Index of Erectile Function (IIEF-5) as a diagnostic tool to study the prevalence of erectile dysfunction. Int J Impot Res. 2002; 14:245-50.
11. Grossi FS, Di Lena S, Barnaba D, et al. Laparoscopic versus open radical retropubic prostatectomy: a case-control study at a single institution. Arch Ital Urol Androl. 2010; 82:109-12.

12. Schuessler WW, Schulam PG, Clayman RV, Kavoussi LR. Laparoscopic radical prostatectomy: initial short-term experience. Urology. 1997; 50:854-7.

13. Türk I, Deger S, Winkelmann B, et al. Laparoscopic radical prostatectomy. Technical aspects and experience with 125 cases. Eur Urol. 2001;40:46-52.

14. Guillonneau B, Vallancien G. Laparoscopic radical prostatectomy: the Montsouris technique. J Urol. 2000;163:1643-9.

15. van Velthoven RFP. Laparoscopic radical prostatectomy: transperitoneal versus retroperitoneal approach: is there an advantage for the patient?. Curr Opin Urol. 2005; 15: 83-8.

16. Porpiglia F, Terrone C, Tarabuzzi R, et al. Transperitoneal versus extraperitoneal laparoscopic radical prostatectomy: experience of a single center. Urology. 2006; 68:376-80.

17. Eden C, King D, Kooiman $G$, et al. Transperitoneal or extraperitoneal laparoscopic radical prostatectomy: does the approach matter? J Urol. 2004; 172:2218-23.

18. Stolzenburg J, Truss M, Bekos A, et al. Does the extraperitoneal laparoscopic approach improve the outcome of radical prostatectomy? Curr Urol Rep. 2004; 5:115-22.

19. Stolzenburg J, Rabenalt R, Do M, et al. Laparoscopic extraperitoneal radical prostatectomy: changes in time and updated results. Actas Urol Esp. 2006; 30:556-66.

20. Magheli A, Busch J, Leva N, et al. Comparison of surgical technique (open vs. laparoscopic) on pathological and long term functional outcomes following radical prostatectomy. BMC Urol. 2014. 7;14:18.

21. Dahl D, Barry M, McGovern F, et al. A prospective study of symptom distress and return to baseline function after open versus laparoscopic radical prostatectomy. J Urol. 2009; 182:956-65.

22. Jurczok A, Zacharias M, Wagner S, et al. Prospective non-randomized evaluation of four mediators of the systemic response after extraperitoneal laparoscopic and open retropubic radical prostatectomy. BJU Int. 2007; 99:1461-6.

23. Jacobsen N, Moore K, Estey E, Voaklander D. Open versus laparoscopic radical prostatectomy: a prospective comparison of postoperative urinary incontinence rates. J Urol. 2007; 177:615-9.

24. Paul A, Ploussard G, Nicolaiew N, et al. Oncologic outcome after extraperitoneal laparoscopic radical prostatectomy: midterm followup of 1115 procedures. Eur Urol. 2010; 57:267-72.

25. Wang $K$, Zhuang $Q, X u R$, et al. Transperitoneal versus extraperitoneal approach in laparoscopic radical prostatectomy: A meta-analysis. Medicine (Baltimore). 2018; 97:e11176.

26. Kallidonis, Panagiotis P, Rai, Bhavan PB, et al. Critical appraisal of literature comparing minimally invasive extraperitoneal and transperitoneal radical prostatectomy: A systematic review and meta-analysis. Arab J Urol. 2017; 15:267-279.

27. Cao L, Yang Z, Qi L, Chen C. Robot-assisted and laparoscopic vs open radical prostatectomy in clinically localized prostate cancer: perioperative, functional, and oncological outcomes: A Systematic review and meta-analysis. Medicine (Baltimore). 2019; 98:e15770.

28. Uchio E, Aslan M, Wells C, et al. Impact of biochemical recurrence in prostate cancer among US veterans. Arch Intern Med. 2010; 170:1390-5. 
29. Chun F, Graefen M, Zacharias M, et al. Anatomic radical retropubic prostatectomy-long-term recurrence-free survival rates for localized prostate cancer World J Urol. 2006; 24:273-80.

30. Hruza M, Bermejo J, Flinspach B, et al. Long-term oncological outcomes after laparoscopic radical prostatectomy. BJU Int. 2013; 111:271-80.

31. Busch J, Stephan C, Herold A, et al. Long-term oncological and continence outcomes after laparoscopic radical prostatectomy: a single-centre experience BJU Int. 2012; 110:E985-90.

32. Martínez-Holguín E, Herranz-Amo F, Mayor de Castro J, et al. Comparison between laparoscopic and open prostatectomy:
Oncological progression analysis. Actas Urol Esp. 2021; 45:139145.

33. Walsh. Radical prostatectomy for localized prostate cancer provides durable cancer control with excellent quality of life: a structured debate. J Urol. 2000; 163:1802-7.

34. Abboudi H, Doyle P, Winkler M. Day case laparoscopic radical prostatectomy. Arch Ital Urol Androl. 2017 ;89:182-185.

35. Gozen AS, Akin Y, Ates $M$, et al. The impact of bladder neck sparing on urinary continence during laparoscopic radical prostatectomy; Results from a high volume centre. Arch Ital Urol Androl. 2017; 89:186-191.

\section{Correspondence}

Francesco Saverio Grossi, MD (Corresponding Author) grossifs@libero.it

Emanuele Utano

Paolo Minafra

Pier Paolo Prontera

Francesco Schiralli

Antonio De Cillis

Evangelista Martinelli

Marco Lattarulo

Meri Luka

Antonio Carrieri

Angelo D'Elia

Department of Urology, SS Annunziata Hospital

Via Francesco Bruno 1, 74121, Taranto (Italy) 